\title{
III-V on-silicon sources for optical interconnect applications
}

\author{
D. Van Thourhout, ${ }^{1,2}$ S. Keyvaninia, ${ }^{1,2}$ M. Tassaert, ${ }^{1,2}$ T. Spuesens, ${ }^{1,2}$ G. Roelkens, ${ }^{1,2}$ B. Tian, ${ }^{1,2}$ Z. Wang, ${ }^{1,2}$ M. \\ Pantouvaki, ${ }^{3}$ J. Van Campenhout, ${ }^{3}$ C. Merckling ${ }^{3}$ \\ ${ }^{1}$ Photonics Research Group, INTEC, Ghent University -imec, Ghent, 9000 Belgium \\ ${ }^{2}$ Center for Nano- and Biophotonics (NB-Photonics), Ghent University, Ghent 9000, Belgium \\ ${ }^{3}$ IMEC, Heverlee, 3001, Belgium \\ email: dries.vanthourhout@ugent.be
}

\begin{abstract}
Optical interconnects require efficient and flexible optical sources. This paper presents results on two technology platforms being developed for realizing these. Integration using wafer bonding technologies is well established now and the focus is on new device types including tunable lasers, multi-wavelength lasers and switching. As an alternative, we also started work on monolithic integration using heteroepitaxy directly on silicon. We here report recent results on low threshold nanowire lasers.
\end{abstract}

Index Terms-III-V on silicon, hetero-epitaxy, wafer bonding, integratd optics, silicon photonics.

\section{INTRODUCTION}

Remarkable progress has been made in the domain of silicon photonics but practical applications still mostly rely on III-V semiconductor based sources, either as an external "power source" or integrated with the chip using relatively standard flip-chip techniques. The only "wafer-scale" approach that seems close to industrial viable at this moment is relying on wafer bonding techniques [1], where the focus now mostly is on improving performance and demonstrating more complex devices (section II). As an alternative we are now also investigating the viability of integrating lasers on (100)-silicon using heteroepitaxy (section III).

\section{INTEGRATION THROUGH WAFERBONDING}

Using an advanced die-to-wafer bonding process [1] we recently showed several novel devices integrated on a silicon photonics platform, including tunable lasers [2], multifrequency lasers [3] and DFB-lasers [4]. Figure 1 shows schematically the integration process with the 2-step taper optimized to couple from the III-V hybrid device towards standard 220nm high silicon waveguides. The picture also shows a longitudinal cross-section with the DFB grating etched in the silicon underlying the III-V.

Figure 2 (top) shows a complex switch, containing thin membrane InP swtiches (MIPS), which can be remotely controlled through adding a label with specific wavelength [5][6]. Figure 2 (middle, bottom) also shows an on-chip low power optical link using a microdisk laser as the source, and operating at 10GBit/s [7][8], realized using our die-to-wafer bonding process.

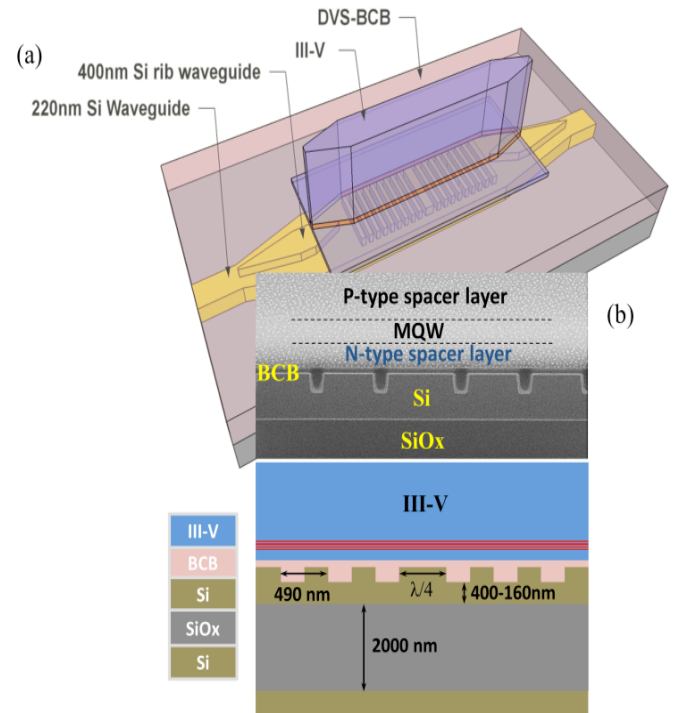

Figure 1 Schematic view of hybrid III-V silicon DFB laser and longitudinal cross-section (from [4]).
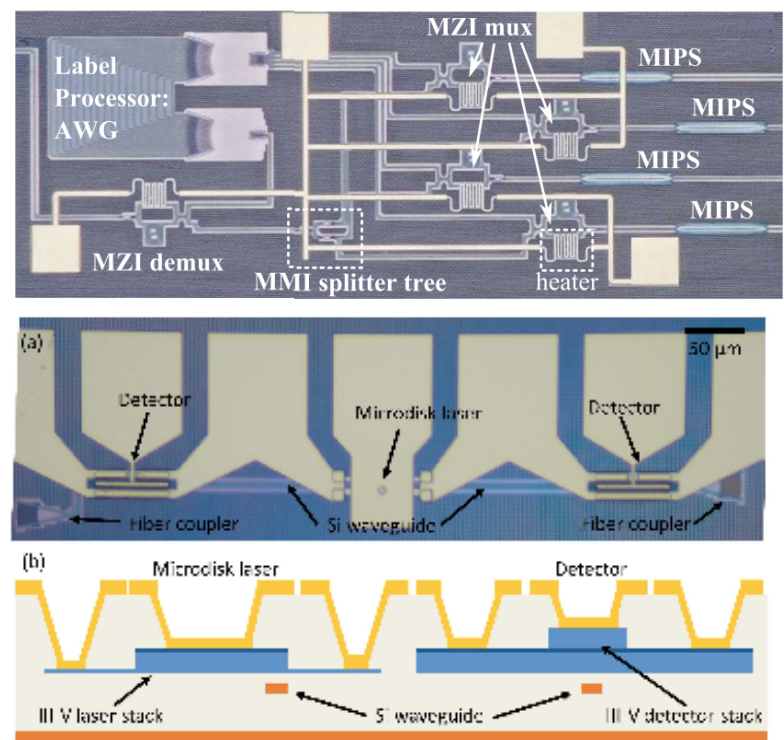

Figure 1 (top) Microscope picture of $1 \mathrm{x} 4$ switch including membrane InP switches (MIPS) [6]. (middle and bottom) Top view (optical microscope) and schematic cross-section of on-chip link consisting of microdisk laser and detector [8]. 


\section{MONOLITHIC INTEGRATION}

While waferbonding allows transferring the highest quality materials, a technique relying on direct epitaxy will ultimately lead to the lowest cost devices. We recently showed lasing from InP-nanowires grown on [001]-oriented silicon using selective area growth in oxide template to limit the defects in the InP layer [9][10][11]. The low threshold obtained testifies of the high quality of the material. Currently we are working towards devices that can be coupled to integrated waveguides. Figure 3 shows a close-upview of one of the nanolasers [11]. Figure 4 shows the photoluminescence spectra below and above threshold.

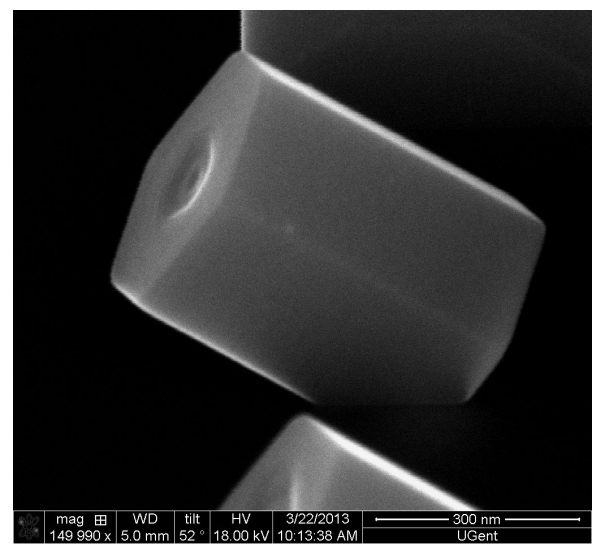

Figure 2 a) InP-nanowire grown on silicon using aspect ratio trapping to suppress defects (from [11])

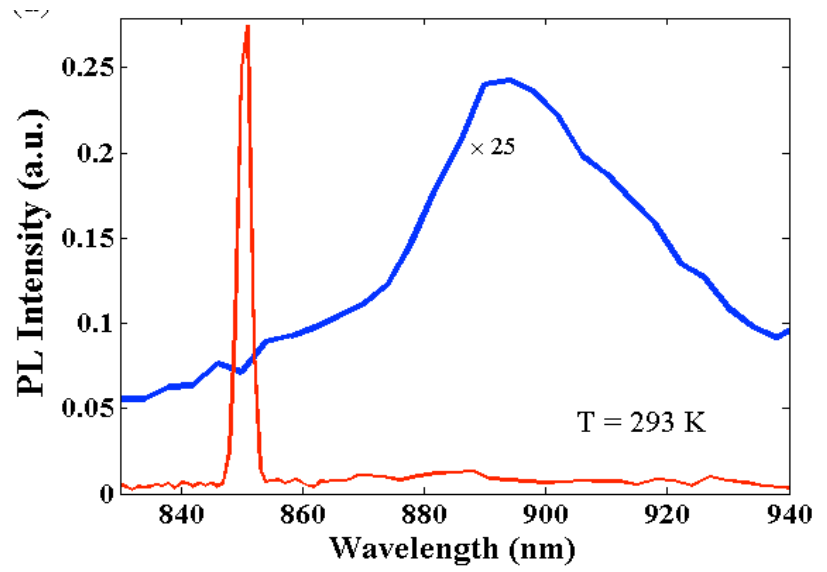

Figure 4 Photoluminescence spectra below (blue) and above (red) laser threshold (from [11])

\section{ACKNOWLEDGMENTS}

We acknowledge support of the EU commission through the ERC-ULPPIC project and support from the Belgium Science office through the IAP photonics@be project. Part of the work was supported by imec's CORE Program on Optical I/O.

\section{REFERENCES}

[1] G. Roelkens, L. Liu, D. Liang, R. Jones, A. Fang, B. Koch, and J. Bowers, "III-V/silicon photonics for on-chip and intra-chip optical interconnects," Laser Photon. Rev., vol. 4, no. 6, pp. 751-779, 2010.

[2] S. Keyvaninia, G. Roelkens, D. Van Thourhout, C. Jany, M. Lamponi, A. Le Liepvre, F. Lelarge, D. Make, G.-H. Duan, D. Bordel, and J.-M. Fedeli, "Demonstration of a heterogeneously integrated III-V/SOI single wavelength tunable laser," Opt. Express, vol. 21, no. 3, p. 3784, Feb. 2013.

[3] S. Keyvaninia, S. Verstuyft, S. Pathak, F. Lelarge, G.-H. Duan, D. Bordel, J.-M. Fedeli, T. de Vries, E. Smalbrugge, E.-J. Geluk, J. Bolk, G. Roelkens, and D. Van Thourhout, "III-V-onsilicon multi-frequency lasers," Opt. Express, vol. 21, no. 11, pp. 13675-13683, 2013.

[4] S. Keyvaninia, D. Van Thourhout, R. Baets, G. Roelkens, G.-H. Duan, F. Lelarge, J.-M. Fedeli, and S. Messaoudene, "Demonstration of a novel III-V-on-Si distributed feedback laser," Opt. Fiber Commun. Conf. Fiber Opt. Eng. Conf. 2013, p. OTh1D.6, 2013.

[5] M. Tassaert, S. Keyvaninia, D. Van Thourhout, W. M. J. Green, Y. Vlasov, and G. Roelkens, "An optically pumped nanophotonic InP/InGaAlAs optical amplifier integrated on a SOI waveguide circuit," Opt. Quantum Electron., vol. 44, no. 12-13, pp. 513-519, Mar. 2012.

[6] M. Tassaert, G. Roelkens, H. Dorren, and O. Raz, "Optically Reconfigurable 1x4 Remote Node Switch for Access Networks,” J. Light. Technol., vol. 32, no. 4, pp. 847-853, 2013.

[7] T. Spuesens, F. Mandorlo, P. Rojo-Romeo, P. Regreny, N. Olivier, J. M. Fedeli, and D. Van Thourhout, "Compact integration of optical sources and detectors on SOI for optical interconnects fabricated in a $200 \mathrm{~mm}$ CMOS pilot line," $J$. Light. Technol., vol. 30, no. 11, pp. 1764-1770, 2012.

[8] T. Spuesens, J. Bauwelinck, P. Regreny, and D. Van Thourhout, "Realization of a Compact Optical Interconnect on Silicon by Heterogeneous Integration of III-V," IEEE Photonics Technol. Lett., vol. 25, no. 14, pp. 1332-1335, Jul. 2013.

[9] M. Paladugu, C. Merckling, R. Loo, O. Richard, H. Bender, J. Dekoster, W. Vandervorst, M. Caymax, and M. Heyns, "Site selective integration of III-V materials on Si for nanoscale logic and photonic devices," Cryst. Growth Des., vol. 12, no. 10, pp. 4696-4702, 2012.

[10] C. Merckling, N. Waldron, S. Jiang, W. Guo, N. Collaert, M. Caymax, E. Vancoille, K. Barla, A. Thean, M. Heyns, and W. Vandervorst, "Heteroepitaxy of InP on Si (001) by selectivearea metal organic vapor-phase epitaxy in sub-50 $\mathrm{nm}$ width trenches: The role of the nucleation layer and the recess engineering," J. Appl. Phys., vol. 115, p. 023710, 2014.

[11] Z. Wang, B. Tian, M. Paladugu, M. Pantouvaki, N. Le Thomas, C. Merckling, W. Guo, J. Dekoster, J. Van Campenhout, P. Absil, and D. Van Thourhout, "Polytypic InP nanolaser monolithically integrated on (001) silicon.," Nano Lett., vol. 13, no. 11, pp. 5063-9, Nov. 2013.

[12] B. Tian, Z. Wang, M. Pantouvaki, W. Guo, M. Clement, and J. Van Campenhout, "InP Nanowire lasers Epitaxially Grown on (001) Silicon 'V-groove' templates," in IPRM 2014, paper Thu-B1-4. 